\title{
Profile of acute severe malaria with hepatopathy
}

\section{Aminul Khan, Gayatri Sarma', Anup K. Das}

Departments of Medicine and ${ }^{1}$ Pharmacology, Assam Medical College Hospital, Dibrugarh, Assam, India

Address for the Correspondence: Prof. Anup K Das, Department of Medicine, Assam Medical College Hospital, Dibrugarh, Assam - 786 002, India.

E-mail: anupkrdas5@gmail.com

\begin{tabular}{|l|}
\hline Access this article online \\
\hline Website: www.jmedph.org \\
\hline DOI: 10.4103/2230-8598.144071 \\
\hline Quick response code: \\
\hline
\end{tabular}

Background: The single most dreaded complication in severe malaria is cerebral malaria, but extracerebral serious complications are becoming frequent in endemic areas, which include hepatic dysfunctions with jaundice. Materials and Methods: This prospective case series study was undertaken to observe the clinical profile in 81 cases of complicated malaria presenting with jaundice out of 344 hospitalized patients diagnosed with acute severe malaria. Liver function tests were assessed and the patients were followed up to 4 weeks. Results: $85 \%$ cases with jaundice had Plasmodium falciparum $(P f)$ infection. Significant findings included a predominantly hemolytic jaundice (mean bilirubin $7.6 \mathrm{mg} \%$, unconjugated $4.83 \mathrm{mg} \%$, conjugated $2.79 \mathrm{mg} \%$ ), raised ALT > AST (mean 101.2 vs.74.7 iu) and a mean prothrombin time of $3 \mathrm{sec}>$ control. Acute renal failure was common (77\%). No residual hepatic dysfunctions were detected in survivors on follow-up. Mortality was $10 \%$, mostly due to delayed diagnosis and associated serious co-morbid conditions. Conclusion: Differentiating fulminant viral hepatitis with multi-organ failure and early treatment of associated complications are crucial to reduce mortality in malaria presenting with jaundice. Hemolytic jaundice with mild and relatively early reversibility of hepatocellular dysfunction usually points towards complicated Pf malaria. Histologically, there is mild hepatic derangement. Acute renal failure is commonly associated. Vivax malaria can also cause hepatic dysfunctions. Mere presence of jaundice does not increase mortality compared to those without jaundice

Key words: Acute severe malaria, complicated malaria, malarial hepatitis, malarial hepatopathy, malarial jaundice

\section{INTRODUCTION}

Malarial epidemics in India have claimed a large number of lives in the last few decades. In Asia, the maximum cases of malaria occur in India. ${ }^{[1]}$ The cause of resurgence of malaria in India may be due to altered ecology, drug resistance and vector resistance to insecticides. ${ }^{[2,3]}$ In the north-east India, Plasmodium falciparum $(P f)$ is the most common cause. Cerebral malaria is the single most important complication leading to mortality ( $\sim 80 \%$ of all malaria cases). But the extracerebral complications like acute renal failure, severe anemia and jaundice can be equally fatal. ${ }^{[4]}$ Often due to their unusual presentations, they are initially misdiagnosed as viral hepatitis or gastroenteritis and can thereby lead to critical delay in initiating specific therapy. ${ }^{[5]}$

The classical clinical spectrum of complicated malaria in India has some differences from those occurring in other parts of world. The north eastern state of Assam, India lies in the endemic belt of $P f$ malaria and almost regular annual epidemics occur here, but there are no data regarding the profile of malaria with jaundice from this part. This hospital-based study was undertaken with the aims of studying the clinical profile of patients with acute malaria and hepatopathy.

\section{MATERIALS AND METHODS}

This was a prospective, single center, 4 year, observational study consisting of series of 344 consecutive cases of malaria, out of which 81 patients (study group, Group A) suffering from acute malaria with jaundice admitted to a Medicine unit of Assam Medical College Hospital, a tertiary center, at Dibrugarh, Assam. Those with acute viral hepatitis were excluded. Those without serum bilirubin $>3 \mathrm{mg} \%$ (Group B, $n$ : 263) were excluded from detailed analysis. 
Malaria was diagnosed by:

a. Positive peripheral thick/thin blood smear (PBS) for malaria parasite, and/or

b. Positive immunofluorescence test employing the QBC blood parasite detection method (EDTA capillaries for blood collection and analysis: Godrej Medical Diagnostic Division, Mumbai 400 079, India).

c. Parachek (commercially available dipstick strip test based on a monoclonal antibody to detect histidine rich protein-2 of Pf by immunochromatographic method: Orchid Biomedical Systems, Goa, India).

A detailed history, clinical examination, and the following investigations were carried out: Liver function tests, prothrombin time, anti-hepatitis A virus (HAV) IgM, anti-hepatitis E virus (HEV) $\mathrm{IgM}$, anti-hepatitis $\mathrm{C}$ virus (HCV), HbsAg, total and differential white blood cells (WBC) count, $\mathrm{Hb}$ gm \%, erythrocyte sedimentation rate (ESR), platelet count, $\mathrm{Hb}$ typing, blood sugar, blood urea, serum creatinine and electrolytes, glucose-6-phosphate dehydrogenase (G-6-PD), urine examination including $\mathrm{Hb}$ and stool for occult blood. Liver biopsy could be done in 10 cases after obtaining consent. Ultrasonogram (USG) was done in all 81 cases at admission.

Blood was examined for malaria parasite on $7^{\text {th }}, 14^{\text {th }}$ and $28^{\text {th }}$ day after treatment for parasite clearance by the above mentioned methods. 17 subjects dropped out after the $1^{\text {st }}$ week.

Follow-up was done with an USG of abdomen ( $28^{\text {th }}$ day), PBS and IF tests for malarial parasites, blood urea, serum creatinine and liver function tests (serum bilirubin, aspartate aminotransferase (AST), alanine transaminase (ALT), alkaline phosphatase). Any biochemical value above upper normal was taken as abnormal.

\section{RESULTS AND OBSERVATION}

Group A consisted of 61 males; 20 non-pregnant females; (mean age $27+/-11.6$ years, range 15-46 years) and had fever for a mean duration of 4.2 days (range $2-7$ days). Group B (n: 263) had a mean age of $36 \pm 9.87$ years [Table 1].

In the present study, hepatic dysfunction manifesting with significant jaundice and raised transaminases were found in 81 (24\%) of 344 cases of acute malaria. Predominantly conjugated hyperbilirubinemia was seen in $19(6 \%)$ cases and unconjugated hyperbilirubinemia in $55(16 \%)$ cases. Raised transaminase $(\mathrm{s})>2 \mathrm{xULN}$ was present in 59 $(22 \%)$ cases whereas $>3 \times$ ULN was seen in $22(13 \%)$.

In Group A, icterus was clinically present in all cases. Of them, 19 $(23 \%)$ had a history of malaria without jaundice and $7(9 \%)$ had history suggestive of viral hepatitis. Majority $(85 \%)$ showed $P f$ infection, and 4 cases $(5 \%)$ had Plasmodium vivax $(P v)$. Mixed infection was observed in 8 cases $(10 \%)$, of which 2 had mixed infection on admission, while the other 6 initially showed $P$ finfection and subsequently found to be positive for $P v$ infection. 72 cases showed asexual ring forms while both gametocytes and trophozoites were present in $9 P f$ cases. All patients had fever, being irregular in 22 patients, and with chill and rigors followed by sweating in 59 cases. Vomiting was present in 51 (63\%) cases. Altered sensorium/restlessness was present in $62(77 \%)$ cases and they had associated acute renal failure (ARF). Some comparative characteristics of the groups A and B are shown in Table 1.

Medical records of $83 \%$ of cases showed them to be inadequately treated with quinine and i.v. fluids outside before referral to our hospital.

Relevant biochemical parameters on admission were: Hemoglobin $8.68 \mathrm{gm} \%+/-1.11$ (range 4.2 to $11 \mathrm{gm} \%$ ), serum bilirubin

\begin{tabular}{|c|c|c|c|}
\hline Parameters studied & Group A ( $n: 81)$ & Group B (n: 263) & $P$ value \\
\hline Age (mean \& SD) yr & $27+/-11.6$ & $36 \pm 9.87$ & Two-tailed $P<0.0001$ \\
\hline Pf & $69(85.18 \%)$ & $207(78.70 \%)$ & \\
\hline$P v$ & $4(4.93 \%)$ & $46(17.49 \%)$ & \\
\hline Mixed $(P f+P v)$ & $8(9.8 \%)$ & $10(3.8 \%)$ & \\
\hline Acute Renal Failure & $62(76.54 \%)$ & $13(4.9 \%)$ & Fisher's Test $<0.0001$ \\
\hline Altered sensorium/Cerebral involvement & $62(76.54 \%)$ & $79(30 \%)$ & Fisher's Test $<0.0001$ \\
\hline Hypoglycemia $<60 \mathrm{mg} / \mathrm{dl}$ & $57(70.37 \%)$ & $41(15.5 \%)$ & Fisher's Test $<0.0001$ \\
\hline Heavy Parasitemia (>5\% infeced RBCs) & $19(23.45 \%)$ & $43(16.34 \%)$ & Fisher's Test equals 0.1850 (not significant) \\
\hline $\mathrm{Hb}$ (mean \& SD) & $8.68 \mathrm{gm} / \mathrm{dl}(1.11)$ & $9.09 \mathrm{gm} / \mathrm{dl}(1.00)$ & Two-tailed $P$ value equals 0.0018 \\
\hline Serum Bilirubin (mean \& SD) & $7.62 \mathrm{mg} / \mathrm{dl}(4.17)$ & $1.31 \mathrm{mg} / \mathrm{dl}(2.01)$ & $<0.0001$ \\
\hline Conj bilirubin (mean \& SD) & $2.79 \mathrm{mg} / \mathrm{dl}(1.81)$ & $0.97 \mathrm{mg} / \mathrm{dl}(0.81)$ & $<0.0001$ \\
\hline Unconj bilirubin (mean \& SD) & $4.83 \mathrm{mg} / \mathrm{dl}(2.58)$ & $1.13 \mathrm{mg} / \mathrm{dl}(1.5)$ & $<0.0001$ \\
\hline AST (mean \& SD) & 74.7 i.u./I (18.06) & 63.6 i.u./I (11.00) & $<0.0001$ \\
\hline ALT (mean \& SD) & 101.28 i.u.// (28.28) & 59.33 i.u.// (15.8) & $<0.0001$ \\
\hline S Alkaline Phosphatase (mean \& SD) & 169 i.u. (14.7) & 190 i.u. (5.9) & $<0.0001$ \\
\hline Prothrombin time (mean \& SD) & $3 \mathrm{sec} .(1.9)>$ control & $1.8 \mathrm{sec} .(1.1)>$ control & $<0.0001$ \\
\hline BI Urea (mean \& SD) & $73.44 \mathrm{mg} / \mathrm{dl}(22.44)$ & $49.28 \mathrm{mg} / \mathrm{dl}(19.72)$ & $<0.0001$ \\
\hline S Creatinine & $3.06 \mathrm{mg} / \mathrm{dl}(1.38)$ & $2.06 \mathrm{mg} / \mathrm{dl}(1.5)$ & $<0.0001$ \\
\hline Mortality & $8(10 \%)$ & $29(11 \%)$ & $P$ value equals 0.8407 not significant \\
\hline
\end{tabular}


$7.62 \mathrm{mg} \%+/-4.17$ (range 2 to $25.9 \mathrm{mg} \%$ ), conjugated bilirubin $2.79 \mathrm{mg} \%+/-1.81$ (range 0.8 to $11 \mathrm{mg} \%$ ), unconjugated bilirubin $4.83 \mathrm{mg}^{\%}+/-2.58$ (range 1.2 to $14.9 \mathrm{mg}^{\%}$ ), AST 74.7+/-18.06 IU/L (range 37 to $116 \mathrm{IU} / \mathrm{L}$ ), ALT $101.28+/-$ $28.28 \mathrm{IU} / \mathrm{L}$ (range 38 to $174 \mathrm{IU} / \mathrm{L}$ ), serum alkaline phosphatase $169 \mathrm{IU} / \mathrm{mL}$ (range 90 to $190 \mathrm{IU}$ ), blood urea $73.44 \mathrm{mg} \%+/-22.44$ (range 32 to $102 \mathrm{mg} \%$ ), serum creatinine $3.06 \mathrm{mg}^{0}+/-1.38$ (range 1.4 to $6.8 \mathrm{mg}^{0}$ ).

A total of 57 cases $(70 \%)$, including those with hyperparasitemia had hypoglycemia $<60 \mathrm{mg} \%$, whereas 2 cases were subsequently found to be diabetic. Acute viral hepatitis serology was negative in all patients. G-6-PD deficiency was found in 9 cases. Hyponatremia $(<130 \mathrm{mmol} / \mathrm{l})$ was present in $44(54.32 \%)$ cases. 6 cases had hemoglobinuria.

Dialysis was done as per Nephrology consultation, at an average of 2.6 days after hospitalization in 62 cases who had ARF [(oliguric $(n: 47)$ or anuric $(n: 14)]$. Total of 56 cases were subjected to peritoneal dialysis (n: 31$)$ and hemodialysis ( $n: 25) .49$ cases needed multiple sittings.

In Group A, hyperparasitemia (Pf) was found in 19 cases. Of them, 8 patients expired within 72 hours of hospitalization (10\% mortality) and had associated serious co-morbidities. None with either G6-PD deficiency or hemoglobinuria expired.

Serious co-morbidities [ $n: 22]$ at presentation [Table 2] were gastro-intestinal (GI) bleeding (2), shock (14), septicemia (3) and gastroenteritis (3).

Liver biopsy did not show any pigment and fibrosis in the liver tissues. Swelling of hepatocytes was universal whereas spotty necrosis was seen in 5 cases. Infiltration with lymphocytes was predominant in 9 cases. Kupffer cell hyperplasia was seen in 2 cases. All cases were biopsied at an average of 4 days of hospitalization while on treatment and none expired.

\section{DISCUSSION}

Disease manifestations and immunity patterns to malaria strongly depend on the level of local malaria transmission; highest risk being in non-immunes, pregnant females and very young children. Age in our study group was significantly lower than Group B, in spite of both groups belonging to endemic Pf belt. Only 2 cases of mixed infection were present on admission and is not uncommon in endemic areas with severe disease. In this study, it was found that $P v$ infection can also present with hepatitis features. Adults in endemic areas may have asymptomatic parasitemia. Although Group A subjects were likely to have been exposed to malaria in the past, still they developed jaundice for the first time. It implies that immunity to malaria is acquired slowly and incompletely despite multiple exposures and is unstable and short lived. ${ }^{[6,7]}$ In malaria, hepatopathy is manifested by hepatomegaly, jaundice/hyperbilirubinemia, and raised aminotransferase levels, ${ }^{[8]}$ but reportedly more common

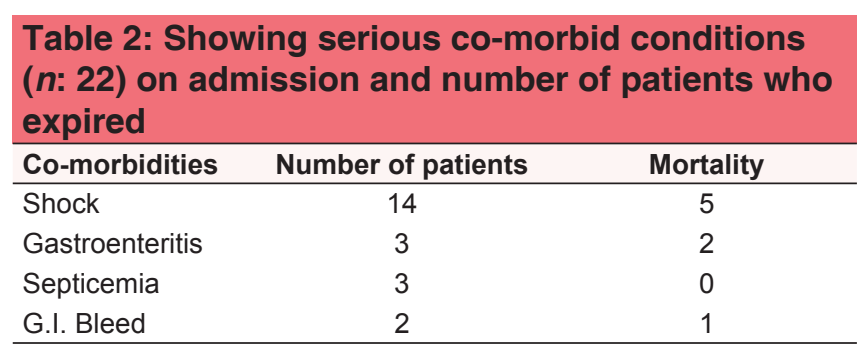

in adults than in children. ${ }^{[1]}$ The term malarial hepatitis implies hepatocellular dysfunction in severe, complicated malaria; but hepatic parenchymal inflammation is reportedly very rare. ${ }^{[9]}$ Hyperbilirubinemia and raised ALT levels to more than 3 times upper limit of normal (ULN) characterizes malarial "hepatitis"[10], whereas others agree that enzymes are usually elevated 2-3 times the normal, ${ }^{[11,12]}$ which is in accordance with our findings where majority of Group A had transaminasemia between 2-3 times ULN, AST being higher than ALT.

According to WHO a bilirubin level $>3 \mathrm{mg}$ is rare in complicated $\mathrm{Pf}$ malaria, which is contrary to our findings. In a study of 50 confirmed cases of $P f$ malaria with jaundice, 18 patients had serum bilirubin $<3 \mathrm{mg} \%$, 20 patients had serum bilirubin between 3-10 $\mathrm{mg} \%$ and only 12 patients had serum bilirubin values of $>10 \mathrm{mg}^{0} .^{[12]}$

The hyperbilirubinemia in malaria can be caused by breakdown of parasitized red blood cells (RBCs), direct hepatic involvement by sequestration, microangiopathic hemolysis and associated septicemia or viral hepatitis. ${ }^{[9]}$ Two biological properties make Pf the most common cause of hepatitis in malaria:

a. Recession of asexual parasites from peripheral circulation to intrahepatic capillaries for later stages of schizogony, and

b. The adhesiveness of the infected RBCs to the vascular endothelium causing hepatic ischemia.

Additionally, local metabolic derangements, cytokines and endogenous pyrogens like Tumor necrosis factor (TNF)-alfa may also contribute. ${ }^{[13-15]}$ Additionally, possible hepatic damage due to malarial infection via oxidative stress-induced mitochondrial pathway of apoptosis due to hydroxyl radical $(\mathrm{OH})$ generation has been proposed. ${ }^{[16]}$ Therefore, multiple factors are responsible for the hepatic dysfunction in severe malarial infection. This may not only be due to direct inflammation of hepatocytes, but a failure of bilirubin excretion due to heavy parasitemia, endotoxemia, ischemia, acidosis or a combination of some or all of these factors. ${ }^{[9]}$

Presence of higher ALT > AST levels in study group suggest some hepatic involvement. The prothrombin time in our series was significantly high in Group A compared with Group B but coagulopathy was absent. In malaria, prothrombin time is usually normal, even in presence of marked elevation of enzymes. ${ }^{[1,12]}$

In adults, the reported incidence of hyperbilirubinemia in severe malaria varies from $32-37 \%$, which is higher compared to our finding. In the majority of the cases, it is predominantly unconjugated 
hyperbilirubinemia. ${ }^{[17,18]}$ In our study group, 68\% had unconjugated hyperbilirubinemia. Therefore, hemolysis can be taken as the major cause of the jaundice in our series but others report that conjugated fraction is dominant in patients with hepatopathy. ${ }^{[12]}$ Also, it can be predominantly conjugated in blackwater fever and very severe disease ${ }^{[19]}$ thereby mimicking hepatocellular injury. This may then be confused with fulminant hepatitis in a background of severe systemic manifestations. ${ }^{[20]}$ But presence of gross hepatocellular failure in malaria, although possible, should always raise the possibility of the presence of concomitant viral hepatitis ${ }^{[1,2]}$ which was absent in our series. Confusion with acute fulminant hepatitis, and possibly the delay in diagnosis led to the mortality ( 6 patients) in early part of the study period. Additionally, a large number of patients were treated inadequately outside. In our opinion, the presence of significant anemia, predominantly unconjugated bilirubinemia in the majority, higher ALT levels (> AST), and with an absolute average enzyme rise, was helpful in distinguishing it from fulminant hepatic failure. One important clinical pointer was persisting fever after the appearance of jaundice, which is uncommon in viral hepatitis.

All our subjects were HbsAg negative, similar to the observation made in another Indian study ${ }^{[21]}$ of $P$ fassociated hepatitis. However, in a case control study involving over 1200 Gambian children with severe malaria, HbsAg carriage was a significant association. ${ }^{[22]}$ This may be related to impaired clearance of liver stage parasites in chronic carriers of HBV infection. The totally opposite finding in our study may have a molecular basis involving different human leukocyte antigen (HLA) status because HLA class II antigens protect against severe malaria. ${ }^{[23]}$

Presence of ARF is generally the most common renal manifestation in complicated malaria ${ }^{[24]}$ as was in our study group. All had altered sensorium. Cerebral malaria, hypoglycemia, hypoxia along with uremia due to renal involvement may all contribute to the encephalopathy in severe $P f$ infection. The high incidence of ARF in our series shows that jaundice may produce an adverse effect on renal function, probably by inducing increased vascular response to catecholamines, plasma renin activity, hyperuricosuria, natriuresis and left ventricular dysfunction as reported. ${ }^{[25]}$ ARF is comparatively rare in children but when it occurs, it may be very severe. ${ }^{[4,26]}$ Hypoglycemia was frequently seen in this study. It is an aviodable cause of mortality and morbidity. In both the groups all expired cases had hypoglycemia. The causes may be inadequate intake/i.v. fluids, hyperparasitemia, vomiting and improper monitoring.

Common histological feature of malarial hepatopathy are acute hepatocellular injury and hemozoin (malarial pigment) deposition, the most consistent findings being reticuloendothelial cell hyperplasia. ${ }^{[27]}$ Pigment can be absent in $25 \%$ cases of severe malaria with jaundice ${ }^{[12]}$ but is usually present in fatal malaria. ${ }^{[28]}$ In few of our cases, liver histology did not show any pigment, possibly due to administration of anti-malarial both in and outside the hospital prior to biopsy. All our cases were relatively stable, did not expire and none had hyperparasitemia. In all cases, cloudy swelling was present with moderate inflammation. In a study of 50 patients with proven $P f$ malaria with jaundice, histo-pathological examination was done in 20 patients with serum bilirubin level 3-10 $\mathrm{mg} \%$. It showed evidence of swollen hepatocytes in 100\%, malarial pigment deposition in $75 \%$, inflammatory infiltrates in $60 \%$, congestion of hepatocytes in $50 \%$ and associated centrizonal necrosis in $25 \%$ of cases. ${ }^{[12]}$ The autopsy of the livers of those with fatal malaria in another Indian study were Kupffer-cell hyperplasia, malarial pigment within the Kupffer cells, and liver-cell necrosis, with portal inflammation, steatosis and cholestasis being observed in some. ${ }^{[28]}$

In Indian studies, mortality in severe malaria with hepatitis and associated other systemic involvement range from $12.4 \%$ to over $50 \% \cdot{ }^{[19,20,26]}$ Mortality in study group was $10 \%$, (two had cerebral malaria) and statistically not significant compared with those without jaundice signifying malarial jaundice in our part is mainly due to hemolysis rather than hepatocellular, even though the liver function tests in Group A was significantly worse than Group B without jaundice. The wide variation in reports of jaundice and hepatocellular dysfunctions in severe malarial infection may be due to the geographic conditions, endemicity of malaria in the region from where the reports have originated, the age groups studied, the epidemic form of infections reported and coexistent viral hepatitis or helmenthic infections endemic to that particular area. ${ }^{[9]}$ The higher tertiary care centers are likely to receive more serious patients and thereby report higher incidence of organ dysfunction like ARF in our series in the case of severe and complicated malaria. It is known that jaundiced malarial patients have more complications like cerebral malaria, acute renal failure, pulmonary edema and shock, ${ }^{[17,29]}$ which actually may be the primary cause of mortality.

At the end of $28^{\text {th }}$ day, $14 \%$ (9 of 64) had abnormal liver functions but were clinically stable and asymptomatic [Figure 1]. Hepatic dysfunction is reversible in all the patients developing malarial hepatopathy, who respond favorably to antimalarial therapy and no residual effects have been documented in survivors. ${ }^{[10,30]}$

In Group B, mortality was slightly higher (11\%) but heavy parasitemia was lesser $(16 \%)$ compared to those in study group.

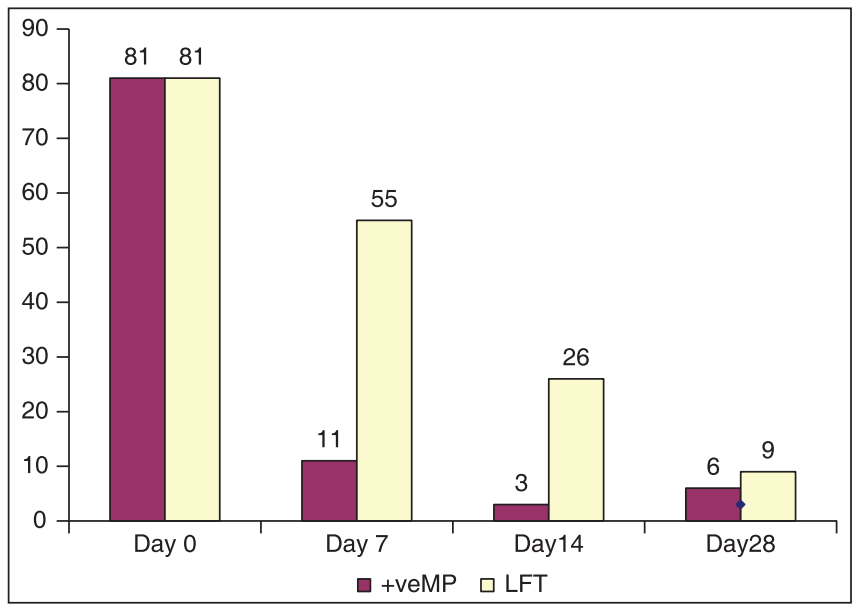

Figure 1: Combination chart showing the altered Liver Function Tests, and Parasitemia on follow- up of patients [Day 0-D7 ( $n$ : 81). D14-D28 ( $n: 64)$ ] 


\begin{tabular}{|c|c|c|}
\hline $\begin{array}{l}\text { Treatment } \\
\text { outcome }\end{array}$ & $\begin{array}{c}\text { Quinine + Artesunate } \\
\text { N: } 47\end{array}$ & $\begin{array}{l}\text { Quinine monotherapy } \\
\qquad: 34\end{array}$ \\
\hline $\mathrm{PF}$ & 43 & 32 \\
\hline$P F+P V$ & 2 & 0 \\
\hline PV & 2 & 2 \\
\hline $\begin{array}{l}\text { Late } \\
\text { Parasitological } \\
\text { Failure (LPF) }\end{array}$ & 2 & 4 \\
\hline $\begin{array}{l}\text { Late Clinical } \\
\text { Failure (LCF) }\end{array}$ & 3 & 6 \\
\hline $\begin{array}{l}\text { Avg Fever } \\
\text { Clearance Days }\end{array}$ & 2.4 & 3.1 \\
\hline
\end{tabular}

\section{Treatment}

Quinine and artesunate were used in the more serious cases compared to the group treated with quinine alone. The group treated with artesunate showed a shorter fever-clearance time and higher parasite clearance [Table 3]. The mortality was nil in quinine-treated group; but, cases with serious co-morbid conditions were treated with the combination therapy where all the deaths occurred. The rationale for the combination therapy was that quinine alone cannot induce rapid clearance of the ring-form-infected RBCs and most importantly, it does not reduce the subsequent cytoadherence, ${ }^{[31,32]}$ whereas artemisinin compounds have these important therapeutic effects - desirable in complicated PF malaria where cytoadherence probably plays the central role in the pathophysiological processes especially in the liver, brain and kidneys. In addition, they have a very rapid in vivo antimalarial activity on the asexual erythrocytic forms ${ }^{[33]}$ thereby quickly bringing down the parasite load. We also presumed that the cases were relatively resistant to quinine since, ${ }^{[34]}$ we found ring forms of the parasite in $89 \%$ of our study group. Moreover, all our cases were treated previously with antimalarials, before presenting to our hospital, and still had a complicated course. We continued quinine because it was given in inadequate dosage outside in the majority. Moreover, the blood concentration of quinine rises progressively to reach the highest serum level in severe disease due to reduced systemic clearance ${ }^{[35,36]}$ and by this way a larger number of mature trophozoites can be destroyed thereby possibly giving a prolonged beneficial effect. We did not find clinically significant toxicity in the artesunate-treated group compared with quinine alone. In general, this combination showed a favorable effect in life-threatening malaria.

\section{CONCLUSION}

Malarial hepatopathy is not ucommon in North-East India, but hemolysis is the major cause of jaundice rather than hepatitis. Associated organ failure and hypoglycemia, and not jaundice, are associated with higher mortality. There may be different mechanism in pathogenesis of malarial jaundice with deranged liver function in our region whose profile varies from other parts in terms of presence of hepatitis. There are some differences in our findings, which were reported from other parts of the world, the most common cause of jaundice being unconjugated hyperbilirubinemia associated with heavy parasitemia, whereas hypoglycemia being very common is not always associated with hyper-parasitemia. We will have to be vigilant to malarial emergencies with extra-cerebral manifestations and treat them adequately. Supportive measures from other allied discipline are needed, as multisystem involvement in Pf infection is common. Interestingly, $P \nu$ infection can also lead to hepato-renal complications as well as cerebral malaria, and mixed infection is not uncommon.

In our region, complicated malaria with hepatopathy (and ARF) are increasing, as observed in the present study and they need to be distinguished from acute fulminant viral hepatitis with type I hepatorenal syndrome. Malaria shows, as compared to viral hepatitis, ALT > AST (usually 2-3xULN), predominantly unconjugated bilirubinemia, mildly elevated prothrobin time, and the associated renal failure is correctable rapidly with early dialysis and specific antimalarials given in adequate dosage. The diagnosis is confirmed by a positive PBS and newer sensitive tests. A high index of suspicion is warranted especially in cases presenting with high fever and jaundice, as the mortality can increase with delay in starting antimalarials. Deranged liver functions are significantly common in severe malarial infection; however, histologically severe hepatic inflammation is probably not a major factor in our region. The hepatic dysfunctions recover without any persistent short-term hepatic complications. However, mortality is not different from those without hepatopathy.

\section{REFERENCES}

1. Gujar PN, Gujar KN, Parale M. Falciparum malaria. Q Med Rev 1998;49:2, 25.

2. In: Misra NP, editor. Malaria: Report of a Scientific group of Indian College of Physicians Technical Report Series II. Indian College of Physicians, Mumbai; 1996;1:23-6.

3. Khera AK, Jain DC, Datta KK. Profile of epidemic emergencies in India during 1991-95. J Commun Dis 1996;28:129-38.

4. Bag S, Samal GC, Deep N, Patra UC, Nayak M, Meher LK. Complicated falciparum malaria. Indian Pediatr 1994;31:821-5.

5. Kyriacou DN, Spira AM, Talan DA, Mabey DC. Emergency department presentation and misdiagnosis of imported falciparum malaria. Ann Emerg Med 1996;27:696-9.

6. Playfair JH. Immunity to malaria. Br Med Bull 1982;38:153-9.

7. Kaplan MJ, Burns JM, Vaidya AB, Webster HK, Weidanz WP. Immunology and molecular biology of parasitic infection. In: Warren KS, editor. Malaria. $3^{\text {rd }}$ ed. Blackwell Scientific Publications; 1993.1:302-35.

8. Botella-de-Maglia J, Espacio-Casanovas A. Severe and complicated malaria. Report of six cases. Rev Clin Esp 1998;198:509-13.

9. Anand AC, Puri P. Jaundice in Malaria. J Gastroenterol Hepatol 2005;20:1322-32.

10. Anand AC, Ramji C, Narula AS, Singh W. Malarial hepatitis: A heterogeneous syndrome? Natl Med J India 1992;5:59-62.

11. Devarbhavi H, Alvares JF, Kumar KS. Severe falciparum malaria simulating fulminant hepatic failure. Mayo Clin Proc 2005;80:355-8.

12. Kochar DK, Singh P, Agarwal P, Kochar SK, Pokharna R, Sareen PK. Malarial hepatitis. J Assoc Physics India 2003;51:1069-72.

13. In: Misra NP, editor. Malaria: Report of a scientific group of Indian college of physicians. Technical Report Series II. Indian College of Physicians, Mumbai 1996;4:32-4.

14. Clark IA, Chaudhri G, Cowden WB. Roles of tumour necrosis factor in the illness and pathology of malaria. Trans R Soc Trop Med Hyg 1989;83:436-40.

15. Phillips RE, Warrel DA. The pathophysiology of severe falciparum malaria. Parasitol Today 1986;2:271-82. 
16. Guha M, Kumar S, Dubey V, Maity P, Bandopadhayay U. Apoptosis in liver during malaria: Role of oxidative stress and implication of mitochondrial pathway. FASEB J 2006;20:1224-6.

17. Wilairatana P, Looareesuwan S, Charoenlarp P. Liver profile changes and complications in jaundiced patients with falciparum malaria. Trop Med Parasitol 1994;45:298-302.

18. Harris VK, Richard VS, Mathai E, Sitaram U, Kumar KV, Cherian AM, et al. A study of clinical profile of falciparum malaria in a tertiary care hospital in south India. Indian J Malariol 2001;38:19-24.

19. Ahsan T, Rab SM, Shekhani MS. Falciparum malaria or fulminant hepatic failure? J Pak Med Assoc 1993;43:206-8.

20. Srivastava A, Khanduri A, Lakhtakia S, Pandey R, Chaudhri G. Falciparum malaria with acute liver failure. Trop Gastroenterol 1996;17:172-4.

21. Gupta UC, Kataria ML. Plasmodium falciparum hepatitis during malaria epidemic. J Assoc Physicians India 1993;41:292.

22. Thursz MR, Kwiatkowski D, Torok ME, Allsopp CE, Greenwood BM, Whittle $\mathrm{HC}$, et al. Association of hepatitis B surface antigen carriage with severe malaria in Gambian children. Nat Med 1995;1:374-5.

23. Hill AV, Allsopp CE, Kwiatkowski D, Anstey NM, Twumasi P, Rowe PA, et al. Common West African HLA antigens are associated with protection from severe malaria. Nature 1991;352:595-600.

24. Sitprija V. Nephropathy in falciparum malaria. Kidney Int 1988;34:867-77.

25. Bloom D, McCalden TA. Effect of jaundiced plasma on vascular sensitivity to noradrenalin. Kidney Int 1975;8:149-57.

26. Sarkar S, Prakash D, Marwaha RK, Garewal G, Kumar L, Singhi S, et al. Acute intravascular haemolysis in glucose-6-phosphate dehydrogenase deficiency. Ann Trop Paediatr 1993;13:391-4.

27. Chawla LS, Sidhu G, Sabharwal BD, Bhatia KL, Sood A. Jaundice in plasmodium falciparum. J Assoc Physics India 1989;37:390-1.

28. Rupani $A B$, Amarapurkar AD. Hepatic changes in fatal malaria: An emerging problem. Ann Trop Med Parasitol 2009;103:119-27.

29. Desai SP, Vora IM, Pathak AM, Bhalerao M. Plasmodium falciparum malaria — a diagnostic dilemma. Indian J Pathol Microbiol 1996;39:477-9.

30. Ghoda MK. Falciparum hepatopathy: A reversible and transient involvement of liver in falciparum malaria. Trop Gastroenterol 2002;23:70-1.

31. White NJ, Chapman D, Watt G. The effects of multiplication and synchronicity on the vascular distribution of parasites in falciparum malaria. Trans R Soc Trop Med Hyg 1992;86:590-7.

32. White NJ, Krishna S. Treatment of malaria: Some considerations and limitations of the current methods of assessment. Trans R Soc Trop Med Hyg 1989;83:767-77.

33. ter Kuile F, White NJ, Halloway P, Pasvol G, Krishna S. Plasmodium falciparum: In vitro studies of the pharmacodynamic properties of drugs used for the treatment severe malaria. Exp Parasitol 1993;76:85-95.

34. Nicholas JW. Malaria. In: Cook G, editor. Manson's Tropical Diseases, 20th ed. Vol 61. London WB Saunders; 1996. p. 1087-164.

35. Sabcharoen A, Chongsuphajaisiddhi T, Attanath P. Serum quinine concentrations following the initial dose in children with falciparum malaria. Southeast Asian J Trop Med Public Health 1989;13:556-62.

36. White NJ, Looareesuwan S, Warrell DA, Warrell MJ, Bunnag D, Harinasuta T. Quinine pharmacokinetics and toxicity in cerebral and uncomplicated falciparum malaria. Am J Med 1982;73:564-72.

How to cite this article: Khan A, Sarma G, Das AK. Profile of acute severe malaria with hepatopathy. Int J Med Public Health $2014 ; 4: 371-6$

Source of Support: Nil, Conflict of Interest: None declared. 